\title{
Vorbeugen ist besser ... Zur Soziologie der Prävention
}

\author{
Ulrich Bröckling
}

\begin{abstract}
In neun Thesen skizziert der Artikel aus soziologischer Perspektive die spezifische Rationalität und die Technologien vorbeugenden Handelns. Prävention wird dabei beschrieben als übergreifender Modus des Zukunftsmanagements zeitgenössischer Gesellschaften.
\end{abstract}

Keywords: Prävention; Risikosoziologie; Normalismus; Versicherung

In Steven Spielbergs Science-Fiction-Thriller „Minority Report“ verhaftet die Polizei von Washington im Rahmen eines „Precrime“ genannten Modellprogramms im Jahre 2054 prospektive Mörder, bevor diese dazu kommen, ihre Opfer zu töten. In die Lage dazu versetzen sie drei Precognitive, kurz: Precogs, genannte menschliche Wesen, die aufgrund einer gentechnischen Manipulation fähig sind, künftige Morde in traumähnlichen Bildern vorherzusehen und Täter, Opfer und Zeitpunkt der Tat exakt zu bestimmen. Ihre Traumbilder werden mittels einer Ableitung ihrer Gehirnströme auf Bildschirme projiziert und die dort sichtbaren Personen wie Orte durch den Abgleich mit anderen Daten identifiziert, bevor ein Einsatzkommando ausrückt und die Noch-nicht-Mörder festnimmt. Diese werden dann in ein künstliches Koma versetzt und dauerhaft in sargähnlichen Ein-Mann-Silos deponiert. Ein TV-Spot, der für die Ausweitung des Programms auf die gesamten Vereinigten Staaten wirbt, verkündet, dass es „Precrime“ binnen eines Jahres gelungen sei, die Mordrate in Washington auf Null zu reduzieren. Der Spot schließt mit dem Slogan „Denn nur ein Leben in Sicherheit garantiert auch ein Leben in Freiheit“.

Der Film präsentiert mit dieser sich im weiteren Verlauf eher als Schreckensszenario erweisenden Utopie die von den Brechungen des heute Möglichen und Gewollten gereinigte Ratio der Prävention: das Versprechen von Sicherheit, das Ideal totaler gesellschaftlicher Transparenz und das Postulat proaktiver Intervention - gipfelnd in der paradoxen Konstruktion eines Täters ohne Tat. Einige dieser Aspekte beleuchten die folgenden Thesen; in der Summe skizzieren sie so etwas wie die soziale Grammatik der Vorbeugung.

\section{(1) Prävention will nichts schaffen, sie will verhindern.}

Prävention bezeichnet in der grundlegenden Bedeutung des Begriffs ein Handlungsprinzip: Praevenire heißt zuvorkommen. Man tut etwas, bevor ein bestimmtes Ereignis oder ein bestimmter Zustand eintreten, damit diese nicht eintreten oder zumindest der Zeitpunkt ihres Eintretens hinausgeschoben wird oder ihre Folgen begrenzt werden. Vorausgesetzt ist dabei, dass sich erstens aus gegenwärtigen Indikatoren künftige unerwünschte Zustände prognostizieren lassen, dass sich zweitens Anzeichen von Fehlent- 
wicklungen ohne Intervention verschlimmern, folglich drittens frühzeitige Eingriffe die größtmögliche Risikominimierung versprechen und sich die präventiven Interventionen viertens als Hilfe konzeptualisieren lassen.

Der Gegenstandsbereich vorbeugenden Handelns ist offen und nimmt erst im vorbeugenden Zugriff selbst Gestalt an. Indem Prävention, um überhaupt gezielt intervenieren zu können, einen Ausschnitt aus der Wirklichkeit herauslöst und Zusammenhänge zwischen gegenwärtigen Phänomenen und künftigen Ereignissen oder Zuständen postuliert, konstruiert sie ihr eigenes Aktionsfeld. Und da es nichts gibt, was nicht als Bedrohung wahrgenommen oder zur Bedrohung deklariert werden könnte, kann alles zur Zielscheibe präventiver Anstrengungen werden. Ob Karies oder Herzinfarkt, Drogenkonsum oder Jugendgewalt, ob körperliche Deformationen oder psychische Erkrankungen, ob Terroranschläge oder Entwicklung von Massenvernichtungsmitteln - überall lauern Risiken, drohen Krisen und tut folglich Vorbeugung Not.

Prävention folgt dem Prinzip des Latenthaltens: Gesundheit kennt sie nur als Abwesenheit von Krankheit, Sicherheit nur als Ausbleiben von Verbrechen, Frieden nur als Nicht-Krieg. Die Mittel, mit denen sie ihre Ziele erreichen will, sind dagegen ebenso gut repressiver wie produktiver Natur. So wenig ihr Gegenstand a priori festliegt, so gegensätzlich ist auch die Wahl ihrer Interventionsformen und Akteure: Prävention straft und belohnt, droht und ermutigt, schreckt ab und belehrt, sammelt und sondert aus, entzieht Ressourcen und teilt sie $\mathrm{zu}$, installiert technische Kontrollsysteme und nutzt soziale Netzwerke.

Der Unbestimmtheit des Begriffs - das einzige, was die heterogenen Formen präventiven Handelns verbindet, ist ihre zeitliche Struktur: das Zuvorkommen - entsprechen die praktischen Ambivalenzen vorbeugender Interventionen. Im Zeichen von Prävention geschieht Humanstes wie Inhumanstes. Vorbeugung rettet, verlängert und verbessert Leben, sie mindert Leid und Unsicherheit. Vorbeugung kann aber auch gewalttätig, ja mörderisch sein. Sie legitimiert die Todesstrafe ebenso wie die Errichtung von Gefängnissen, Zwangssterilisierungsprogramme ebenso wie die Abtreibung behinderter Föten. Mit dem Vorbeugungsargument werden militärische Erstschläge, die potentiellen Aggressoren zuvorkommen sollen, ebenso gerechtfertigt wie die Liquidierung vermeintlicher „Volksschädlinge“ oder „Klassenfeinde“. Präventionsprogramme gleichen Kreuzzügen; ihre Logik ist die der antizipierenden Säuberung: Gegen welche Übel auch immer die vorbeugenden Maßnahmen antreten, sie sollen eliminiert werden. Auch wenn - anders als in Spielbergs Film - ein endgültiger Sieg den Protagonisten selbst utopisch erscheint und sie sich mit bescheideneren Vorgaben zufrieden geben, als regulative Idee leitet dieses Ziel ihre Praxis. Oft genug liefern Präventionsversprechen nur die Rechtfertigung für Präventionsverbrechen, doch auch jenseits bloß ideologischer Indienstnahme vorbeugender Absichten ist selbst der beste Wille nicht davor gefeit, Schlimmes zu bewirken. Wer dem einen Übel vorbeugt, befördert häufig ein anderes, und der Imperativ der Leidensfreiheit entpuppt sich nicht selten als ein Freibrief für Mitleidslosigkeit.

\section{(2) Prävention versucht die Kontingenz der Zukunft zu bändi- gen.}

Kontingent ist, was unsicher und was veränderbar ist: der Bereich des Möglichen. Nur weil, was sein wird, immer auch anders sein könnte, ist es durch vorbeugendes Handeln beeinflussbar; nur weil das Kontingente immer auch das Bedrohliche, Nicht-Gewollte einschließt, ist vorbeugendes Handeln nötig. Das Kontingente ist „kein offener Letzthori- 
zont des Möglichen überhaupt“" (Makropoulos 1990, 408), sondern immer ein gesellschaftlich gerahmtes Möglichkeitsfeld. In den westlichen Gesellschaften hat sich in der Neuzeit die Kontingenzerfahrung radikalisiert; als unsicher wie veränderbar erscheint seither nicht nur das Handeln selbst, sondern auch der Möglichkeitshorizont, in dem es sich vollzieht. Diese Offenheit wurde jedoch nicht allein als positive Erweiterung der Handlungsoptionen, sondern zugleich als verstörende Entgrenzung erfahren. In dem MaBe, wie für die Unwägbarkeiten des menschlichen Lebens nicht länger ein göttliches $f a$ tum verantwortlich gemacht werden konnte, wurde es möglich und unabweisbar nötig, die bedrohlichen Seiten der Kontingenz aus eigener Kraft unter Kontrolle zu bringen. In diesem Sinne ist Prävention ein modernes Phänomen. Die vorbeugende Kontingenzbewältigung entbehrt jeglichen Bezugs auf Transzendenz und stützt ihre Interventionen auf wissenschaftlich generierte Gewissheiten. Das unterscheidet sie von anderen Formen des Umgangs mit prospektiven Übeln. Vorsorgepraktiken sind vermutlich so alt wie die Menschheit, doch kommen Schutzvorkehrungen gegen Angreifer oder wilde Tiere, das Anlegen von Nahrungsvorräten oder Opferrituale, welche die Götter günstig stimmen und so Schaden abwenden sollen, noch ohne systematische Datenerhebung, Ursachenforschung und Prognostik, ohne minutiöse Steuerungsprogramme und Planbarkeitsutopien aus, wie sie das Zeitalter der Prävention kennzeichnen. Spezifisch modern ist auch die Suggestivkraft des Präventionsgedankens, die Überzeugung, dass vorbeugende Strategien korrektiven und kurativen Maßnahmen grundsätzlich überlegen sind. Weil niemand daran zweifelt, dass Vorbeugen besser ist als Heilen, breiten sich präventive Semantiken und Technologien in nahezu alle Lebensbereiche aus: „prevention of everything - and everything as prevention“"(Billis 1981, 374f.).

\section{(3) Prävention bezieht sich auf Risiken.}

Risiken sind, folgt man Niklas Luhmanns Unterscheidung zwischen Risiko und Gefahr, mögliche künftige Schäden, deren etwaiges Eintreten als Folge eigenen Handelns oder Unterlassens gedeutet, während es bei Gefahren der Umwelt zugerechnet wird (Luhmann 1990, 1991). Ob etwas als Risiko oder als Gefahr erscheint, ist also eine Frage der Selbst- oder Fremdattribution. Zwar kann man sich auch gegen Gefahren wappnen, aber für den, der es tut, verwandeln diese sich insofern in Risiken, als er sie einem Kalkül unterwirft und Eintrittswahrscheinlichkeit beziehungsweise Ausmaß des möglichen künftigen Schadens in Abhängigkeit zum eigenen Handeln oder Unterlassen setzt. Prävention transformiert Gefahren in Risiken, indem sie künftige Zustände an gegenwärtige Entscheidungen koppelt und auf diese Weise Zeit bindet. Wo Vorbeugung möglich erscheint, wie begründet oder unbegründet diese Erwartung auch sein mag, wird es riskant, darauf zu verzichten. Dass ein Haus vom Blitz getroffen wird, ist eine Gefahr; keinen Blitzableiter zu installieren, ein Risiko. Umgekehrt erzeugt präventives Handeln selbst neue Risiken - das Problem jeder Schutzimpfung. Die Entscheidung für oder gegen diese oder jene vorbeugende Maßnahme wird damit zur Abwägung zwischen verschiedenen Risiken und Risikoeinschätzungen. Darin liegt die Brisanz aller Prävention: Entschieden wird in jedem Fall, weil auch Nicht-Entscheiden eine Entscheidung darstellt, aber welche Entscheidung die richtige ist, dafür gibt es keine absoluten Kriterien, sondern bestenfalls Wahrscheinlichkeitsaussagen. Und weil die Zukunft nun einmal kontingent ist, kann Prävention sich auch als ganz unnötig erweisen. Luhmann hat auch das in der ihm eigenen Lakonik auf eine Formel gebracht: „Man jagt sich Tag für Tag durch den Wald, um gesund zu bleiben, und stürzt dann mit dem Flugzeug ab“(Luhmann 1991, 39). 
Risiko ist all das, so ließe sich zuspitzen, wogegen sich vorbeugende oder schadensausgleichende Maßnahmen treffen lassen. Kein „objektives“ Merkmal der Realität also, aber ebenso wenig nur ein soziales Konstrukt oder eine bloß subjektive Einschätzung, sondern ein „Rationalitätsschema“, eine Art und Weise, bestimmte Elemente der Realität zu ordnen, sie kalkulierbar zu machen und gezielt auf sie einzuwirken (Ewald 1993; Dean 1998). Anders ausgedrückt: Risiken sind gebunden an die Existenz und den Einsatz von Risikotechnologien, und verschiedenen Risikotechnologien entsprechen verschiedene Risikotypen.

Zwei grundsätzliche Strategien des Umgangs mit Risiken beziehungsweise der Kontingenzkontrolle lassen sich unterscheiden: Kontingenz kann zum einen bewältigt, der Bereich des Möglichen also durch vorbeugende Reglementierungen eingeschränkt werden. Das Ideal ist hier Risikovermeidung. Kontingenz kann zum anderen „bewirtschaftet", der Möglichkeitshorizont also genutzt und erweitert, seine bedrohlichen Seiten aber durch geeignete Sicherheitsvorkehrungen aufgefangen werden. Das Ideal ist hier Risikomanagement (Makropoulos 1990, 417f.). Man kann das Eintreten möglicher künftiger unerwünschter Ereignisse zu verhindern suchen, oder man kann Vorsorge treffen, dass im Falle ihres Eintretens der Schaden kompensiert wird - etwa durch Abschluss einer Versicherung. Beide Strategien schließen einander nicht aus, sondern verhalten sich komplementär und treten in vielfachen Kombinationen auf. Die Grenzen der einen fungieren als Rechtfertigung der jeweils anderen: Weil auch noch so umfassende Prävention keine absolute Sicherheit garantieren kann, werden Rest-Risiken versicherungsförmig abgefedert; weil Versicherungen Risiken kapitalisieren, sind sie darauf bedacht, Zahl und Ausmaß der Schadensfälle durch vorbeugende Maßnahmen zu begrenzen. So machen Versicherungsgesellschaften ihre Leistungen von der Erfüllung bestimmter präventiver Obliegenheiten abhängig - ohne jährlichen Zahnarztbesuch wird beispielsweise nur ein geringerer Anteil der Kosten für Zahnersatz übernommen. Umgekehrt werden auch regelmäßige Vorsorgeuntersuchungen kaum jemanden veranlassen, auf den Abschluss einer Kranken- oder Unfallversicherung zu verzichten. Historisch war der Aufstieg des Assekuranzwesens begleitet von kritischen Stimmen, die den Versicherungen vorwarfen, sie unterhöhlten das Prinzip individueller Daseinsvorsorge und verführten zu Leichtsinn und Verantwortungslosigkeit. Regelmäßige Beitragszahlung ersetze die Tugend vorbeugender Umsicht. Die Fürsprecher der Versicherung wiederum hielten dagegen, dass der Einzelne sich gegen viele Risiken der industriellen Gesellschaft - Musterbeispiel war der Arbeitsunfall - auch durch noch so vorsichtiges Verhalten nicht schützen könne. Deshalb müssten die Risiken sozialisiert und die Schadensfolgen gemeinschaftlich aufgefangen werden (Ewald 1989, 1991, 1993; Defert 1991).

Mit der Versicherung teilt die Prävention das probabilistische Kalkül. Während die Assekuranz den bereits eingetretenen Schaden durch Geldleistungen ausgleicht und die Mittel dazu durch Prämienzahlungen ihrer Mitglieder aufbringt, soll Prävention im Vorfeld verhindern, dass der Schadens- und damit auch der Versicherungsfall überhaupt eintritt. Die Formel Risiko = Schadensausma 3 x Eintrittswahrscheinlichkeit dient der einen zur Berechnung ihrer Prämien, der anderen zur rationalen Planung ihrer Interventionen, die an einer oder an beiden Variablen zugleich ansetzen. Anders als die Prävention bildet die Versicherung jedoch eine einheitliche Risikotechnologie und vereinigt ihre Mitglieder durch das Prinzip der Solidarhaftung zu einem höchst wirksamen Gesellschaftsvertrag.

Im Unterschied dazu faltet sich Prävention auf in ein komplexes Bündel heterogener Technologien, die sich hinsichtlich ihrer Reichweite, ihres Zeithorizonts, ihrer Angriffspunkte und Wirkmechanismen sowie der beteiligten Akteure unterscheiden und ergänzen: Verhaltens- steht neben Verhältnis-, Spezial- neben Generalprävention, individuum- 
zentrierte konkurrieren mit risikogruppen- oder bevölkerungsbezogenen Ansätzen, Zwangsmaßnahmen mit Aufklärungskampagnen. Im Bereich der Gesundheitsvorsorge soll Primärprävention die Zahl der Neuerkrankungen verringern, Sekundärprävention die Krankheitsdauer verkürzen und Chronifizierungsprozessen vorbeugen, Tertiärprävention schließlich soll Folgeschäden verhindern oder begrenzen (Caplan 1964).

Die Mittel der Prävention sind so verschieden wie die Übel, denen vorgebeugt werden soll: Ihrer Funktionsweise nach lassen sich die Technologien der Vorbeugung klassifizieren in Maßnahmen zur Früherkennung (z.B. Vorsorgeuntersuchungen; Pränataldiagnostik; militärische Aufklärungssysteme), der Expositionsprophylaxe (z.B. Rauchverbote; Wasser- und Abfallhygiene; Wegsperren „gefährlicher Individuen“; Umwelt- und Arbeitsschutzgesetzgebung) und der Dispositionsprophylaxe, die sich wiederum in Maßnahmen zur Verhaltensmodifikation (z.B. pädagogische Interventionen, strafrechtliche Sanktionen, Empowerment, Strategien des Selbstmanagements) und zur Immunisierung (z.B. Impfprogramme, Selbstverteidigungskurse) gliedern. Früherkennung funktioniert als Risiko-Detektor und isoliert Risikofaktoren und -gruppen; Expositionsprophylaxe schirmt gegen potentielle Bedrohungen ab oder eliminiert deren Ursachen; Dispositionsprophylaxe stärkt die Abwehrkräfte.

\section{(4) Prävention ist ein unabschließbares Projekt.}

Sicherheit ist, anders als der Werbespot in Spielbergs Film suggeriert („,Denn nur ein Leben in Sicherheit garantiert auch ein Leben in Freiheit."), ein unerreichbares Ziel. Gleichwohl bilden Sicherheitsbedürfnisse den Antrieb und markieren Sicherheitsfiktionen den Fluchtpunkt aller präventiven Anstrengungen. Das verleiht diesen den Charakter des Unabschließbaren: Vorbeugen kann man nie genug und nie früh genug.

Prävention konstituiert damit eine Anthropologie im Gerundivum. Sie bestimmt „den Menschen" als ein zu schützendes und zu optimierendes Wesen. Sie sucht weder nach Universalien menschlicher Existenz, noch verwirft sie deren Möglichkeit, sondern übersetzt die Frage nach der conditio humana in die praktische Aufgabe, „Defizitmenschen“ zu verhindern und „Voll-“ bzw. „Normalmenschen“ zu schaffen. Den an die Vorbeugungspraktiken geknüpften Sicherheitsversprechen liegt das Bild einer existentiell gefährdeten und gleichermaßen schutzbedürftigen wie zur vernunftmäßigen, d.h. risikominimierenden Lebensführung fähigen Spezies zugrunde. Der unabschließbare Perfektibilisierungsdruck wiederum verpflichtet die Menschen auf das Diktat des Komparativs und bestimmt sie als stets nur vorläufiges Ergebnis von Regimen der (Selbst-)Rationalisierung.

\section{(5) Prävention bedarf eines „Willens zum Wissen“.}

Wer die Wahrscheinlichkeit des Eintretens oder das Ausmaß von Schadensvorfällen minimieren will, muss die Bedingungen kennen, die sie hervorbringen: ohne Ätiologie keine Prognostik, ohne Prognostik keine Prävention. Vorbeugung impliziert daher systematische Wissensproduktion. „Voir pour savoir, savoir pour prévoir, prévoir pour prévenir", heißt es schon bei Comte. Biologische Prozesse, menschliches Verhalten und erst recht soziale Phänomene lassen sich jedoch in den meisten Fällen nicht auf eindeutige Ursache-Wirkungs-Zusammenhänge reduzieren, und selbst wenn Kausalerklärungen Plausibilität beanspruchen können, gilt das nur a posteriori. In Bezug auf die Zukunft sind dagegen nur Wahrscheinlichkeitsaussagen möglich. 
Der präventive Blick generalisiert deshalb den Verdacht und sucht Indizien aufzuspüren, die auf künftige Übel hindeuten und an denen die vorbeugenden Maßnahmen ansetzen können. Das kann der Erreger sein, von dem eine Infektionsgefahr ausgeht, oder das geschwächte Immunsystem, das jenem keinen ausreichenden Widerstand entgegenzusetzen vermag. Das kann ein überschrittener Grenzwert sein oder ein individuelles Risikoverhalten, ein genetischer Defekt oder ein belastendes Sozialmilieu. Das kann schließlich die sprichwörtliche Gelegenheit sein, die Diebe schafft, oder die inzwischen kaum weniger sprichwörtlichen broken windows, die, nicht repariert, die Zahl der Regelverstöße in einem Stadtviertel ansteigen lassen (Wilson/Kelling 1982). Die ätiologische Forschung isoliert und korreliert Risikofaktoren, ohne diese jemals vollständig erfassen zu können. Das Präventionswissen bleibt stets lückenhaft und erheischt weitere Forschung. Wer vorbeugen will, weiß nie genug.

Freilich gibt es auch eine Präventivwirkung des Nichtwissens: Wie Heinrich Popitz für den Bereich des Rechts gezeigt hat, ist „eine totale Verhaltenstransparenz menschlicher Gesellschaften“ ebenso unmöglich wie „ein Normensystem, das die Entdeckung aller Normbrüche aushalten würde“, oder ein Sanktionssystem, das „mit allen Normbrüchen, die passieren, fertig werden müßte“ (Popitz 1968, 18). Nicht nur die verhängten Sanktionen, sondern auch die nicht entdeckten und folglich auch nicht geahndeten Normverletzungen, so sein Argument, stabilisieren die Rechtsordnung, weil sie diese von der Überforderung durch einen lückenlosen Sanktionszwang entlasten, ohne den Geltungsanspruch der Normen preiszugeben. Popitz' Nachweis des sozialen Nutzens der Dunkelziffer zeigt die Schwächen funktionalistischer Straftheorien, widerlegt jedoch nicht die These von der Unabschließbarkeit des präventiven Willens zum Wissen: Die Dunkelziffer ist das, was nach den polizeilichen Anstrengungen zur Verbrechensverhütung und -aufklärung übrig bleibt. Würde aus dieser relativen Schranke des Wissens ein vorsätzliches Nichtwissenwollen, verkehrte sich der präventive Effekt in sein Gegenteil, weil mit der Sanktionsdrohung auch die Norm selbst entwertet würde.

\section{(6) Prävention normalisiert.}

Zum Risikosignal und Ausgangspunkt präventiven Handelns kann letztlich alles werden, was von Sollwerten abweicht oder, besser noch: was sich als Vorzeichen solcher Abweichungen identifizieren lässt. Ob dabei soziale oder biologische Normen oder statistische Mittelwerte als Richtschnur dienen, ist zunächst gleichgültig, entscheidend ist, dass überhaupt Normen bestimmt und Normalverteilungen erhoben worden sind, auf welche die vorbeugenden Interventionen dann geeicht werden. ${ }^{1}$ In dem Maße jedoch, in dem Prävention Normbrüche, Pathologien und Abweichungen von Durchschnittswerten glei-

1 Georges Canguilhem (1974, 166f.) hat darauf hingewiesen, dass logisch zwar die Abweichung auf die Definition des Normalen folgt, sie ist dessen Negation, dass tatsächlich aber die Abweichung das Vorgängige darstellt. Es ist, so Canguilhem, die geschichtliche Priorität des späteren Anormalen, welche eine norm(alis)ierende Intention wachruft. Das Normale ist das Resultat der Durchführung eines norm(alis)ierenden Plans, z.B. eines Präventionsprogramms. Um gezielte vorbeugende Maßnahmen treffen zu können, muss so etwas wie ein Risikobewusstsein vorhanden sein, das dann die Definition von Normen und Normalfeldern vorantreibt und diffuse Störungen in präzise bestimmbare Devianzen oder Pathologien verwandelt. 
chermaßen und mit Hilfe der gleichen normalisierenden Verfahren traktiert, wird sie selbst zum Motor des „Normalismus“(Link 1997).

Um eine diffuse, räumlich wie zeitlich weit verstreute Menge von Menschen in eine Population zu verwandeln, die dann zum Adressaten von Kontroll- und Steuerungsmaßnahmen werden kann, bedarf es Verfahren, welche die Regelmäßigkeiten ihrer LebensäuBerungen sichtbar machen und deren Einflussfaktoren ermitteln. Erst die systematische Erhebung und statistische Aggregation von Daten lässt jene „sozialen Tatbestände“ (Durkheim 1895) emergieren, die eine sich als „Soziale Physik“ (Quetelet 1869) verstehende Soziologie beschreibt und für vorbeugende Eingriffe aufbereitet. Die Entdeckung der eigenständigen Sphäre des Sozialen bildet die epistemische Grundlage aller Präventionsprogramme. Selbst wo die Interventionen beim Individuum ansetzen, bleiben sie durch ihre Orientierung an Normalwerten rückgebunden an die Gesamtpopulation, aus welcher die statistische Fiktion des Durchschnittsmenschen destilliert wurde. Die Geschichte der Prävention ist daher untrennbar verbunden mit der Geschichte der Datenerhebung und -verarbeitung, der Statistik und Wahrscheinlichkeitsrechnung.

Wenn die Kriminalprävention sich beispielsweise nicht mehr darauf beschränkt, Gesetzesbrecher zu bestrafen, um diese wie auch alle übrigen Gesellschaftsmitglieder von künftigen Straftaten abzuhalten, sondern Kriminalitätsraten berechnet und kriminogene Faktoren (Gelegenheitsstrukturen) herauspräpariert, um dann auf diese einzuwirken, so überlagern normalistische Steuerungsmechanismen die normativen Reglementierungen. Umgekehrt wird die Normalität selbst normativ aufgeladen. Das Mittel- wird zum Idealmaß, während Abweichungen vom Durchschnitt Risikogruppen definieren und Präventionsbedarf signalisieren. Die Anziehungskraft vorbeugenden Handelns beruht nicht zuletzt darauf, dass sie die Denormalisierungsangst - vielleicht die Grundangst der Moderne zugleich mobilisiert und zu bewältigen verspricht. „Bin ich noch normal?“, das ist die Frage, die den auf vorbeugende Sorge um sich selbst getrimmten Homo praeventionalis umtreibt. Praktisch funktioniert Vorbeugung als Anpassung und Selbstanpassung an Normalitätsstandards, die damit den Status sozialer Normen erlangen. „Keeping the normals normal“" (Reiwald 1948, 106), lautet ihre Maxime. Und weil die Normalitätsnormen selbst flexibel sind, kann die vorbeugende Anpassung nicht endgültig sein: Wer vorbeugen will, darf niemals aufhören.

\section{(7) Prävention totalisiert und individualisiert.}

Prävention richtet sich gleichermaßen an alle wie an jeden Einzelnen. Deshalb werden die Methoden der Formalisierung und Quantifizierung flankiert von qualitativhermeneutischen Verfahren, die subjektive Sinnwelten und Handlungsmuster ausdeuten. So generieren Verhaltensbeobachtungen, Interviews, Tagebücher oder Krankengeschichten ein individualisierendes Wissen, das Vorbeugung an die Fähigkeit zur Introspektion und den Aufbau von Selbststeuerungspotentialen koppelt. Die vom Ideal der Mathematisierbarkeit getragenen messenden Verfahren und die gerade auf das nicht Mathematisierbare abzielenden phänomenologisch-verstehenden Zugänge stehen sich dabei nicht antagonistisch gegenüber, sondern sind in einem komplexen Verhältnis wechselseitiger Ver- 
weisungen und Verbindungen aufeinander bezogen. ${ }^{2}$ So bilden epidemiologische Erhebungen auf der einen, Individualdiagnostik und Case-management auf der anderen Seite die beiden unverzichtbaren Säulen der Gesundheitsvorsorge. Der Zweigleisigkeit präventiver Wissensproduktion korrespondiert die Gleichzeitigkeit von Dezentrierung und Rezentrierung des Subjekts in der vorbeugenden Praxis: Einerseits ist Prävention, wie der französische Soziologe Robert Castel schreibt, ,,mit der Auflösung des Begriffs des Subjekts oder des konkreten Individuums verbunden, der durch einen Komplex von Faktoren, die Risikofaktoren, ersetzt wird“ (Castel 1983, 51). Andererseits machen vorbeugende Strategien gerade die Seite des Subjekts stark und inaugurieren es als selbstverantwortlichen und kompetenten Agenten seines eigenen Lebens.

\section{(8) Prävention ist ein Handeln in Machtrelationen.}

Wer vorbeugen will, muss nicht nur wissen, was zu tun ist, sondern muss es auch durchsetzen können. Prävention impliziert die Fähigkeit, Verhalten zu steuern und Verhältnisse zu ändern, gleich ob diese Fähigkeit sich auf Sanktionsgewalt oder Überzeugungskraft, auf technische Apparaturen oder soziale Arrangements stützt. Die vorbeugenden Maßnahmen mögen autoritär die individuelle Freiheit beschneiden oder - als Selbstkontrolle - die Autonomie des Individuums bestätigen, ihre Legitimität und Attraktivität beziehen sie aus dem Versprechen, mögliche Schäden abzuwehren. Indem ihr notwendig das Wissen, die Werturteile und damit die Machtkonstellationen der Gegenwart eingeschrieben sind, projiziert Prävention die Gegenwart normativ auf die Zukunft. Sie ist konservativ, selbst wenn sie das Leben von Individuen, Gruppen oder ganzen Populationen nachhaltig verändert.

Wo Macht ausgeübt wird, gibt es auch Widerstand: Prävention ist stets konfrontiert mit Gegenkräften, die ihre Anstrengungen unterlaufen, bremsen oder blockieren, und sie

2 David Matza $(1973,22)$ konstatiert demgegenüber einen Gegensatz von Präventions- und Verstehensperspektive, zumindest soweit es um die Untersuchung abweichenden Verhaltens geht: „Eine grundlegende Schwierigkeit der Präventionsperspektive besteht darin, dass sie systematisch die Fähigkeit zur Einfühlung und zum Begreifen der Untersuchungsgegenstände stört. Nur mit der Perspektive des Verstehens können die Struktur der sozialen Verhaltensmuster und die vielfältigen Nuancen menschlichen Eingehens auf diese Muster erfasst und analysiert werden. Ohne Verstehen und Einfühlung können wir vielleicht die offen zutage liegenden Fakten in bezug auf ein bestimmtes Phänomen sammeln und die darauf gerichteten Maßnahmen kritisieren, aber es wird uns nicht gelingen, in zureichender Tiefe seinen Sinn für die beteiligten Subjekte und seine Stellung im gesamtgesellschaftlichen Zusammenhang zu betrachten. [...] Der Präventionsstandpunkt hindert überhaupt daran, das abweichende Phänomen richtig in den Blick zu bekommen, da er von dem Ziel bestimmt und motiviert wird, es auszumerzen“. Die Opposition ist freilich eher als ein historisches Durchgangsstadium zu begreifen: Matzas Vorwurf bezieht sich auf eine frühe Phase US-amerikanischer Devianzforschung, als Soziologie, Sozialarbeit und Sozialreform eng miteinander verbunden waren und die soziologische Forschung ihrem Selbstverständnis nach unmittelbar zur Heilung bzw. Vorbeugung sozialer Pathologien beitragen wollte. Damit legte sie ihren Untersuchungen nicht nur die dominierenden gesellschaftlichen Normvorstellungen als Maßstab zugrunde und verstellte sich die Möglichkeit, abweichendes Verhalten aus der Akteursperspektive zu beschreiben, die methodische Voreingenommenheit begrenzte vielmehr auch die Wirksamkeit der darauf aufbauenden Präventionskonzepte. Die späteren Studien, etwa der Chicago School, die sich den Devianzphänomenen mit ethnographischem Blick näherten, mündeten nicht mehr unmittelbar in sozialreformerische Programme, aber gerade diese Integration der „Verstehensperspektive“ und die damit verbundene Lockerung des Verhältnisses von Forschung und Anwendung ermöglichte die Verfeinerung und Ausdifferenzierung präventiver Maßnahmen. 
gewinnt erst in der Auseinandersetzung mit diesen Kontur. Von den lieb gewonnenen, aber riskanten Gewohnheiten, an deren Schwerkraft Aufklärungskampagnen ebenso scheitern wie Verbote, bis zu politischen Kontroversen, in denen präventive mit nichtpräventiven und verschiedene präventive Optionen miteinander konkurrieren - immer operiert das vorbeugende Handeln in einer komplexen strategischen Konstellation, in der Kräfteverhältnisse abzuschätzen, Allianzen zu schließen oder aufzukündigen, taktische Festlegungen zu treffen und bei jedem Schritt die Operationen der anderen beteiligten Akteure zu berücksichtigen sind.

\section{(9) Prävention ist gekoppelt an Kosten-Nutzen-Kalküle.}

Ihre Legitimation bezieht Prävention aus dem Versprechen, die gewünschten Effekte mit weniger Aufwand beziehungsweise mit dem gleichen Aufwand größere Effekte zu erzielen als therapeutische Maßnahmen, die Sanktionierung von Abweichungen oder Schadensausgleich. Vorbeugen ist besser, nicht zuletzt weil es billiger ist. Aber auch Prävention hat ihren Preis und gerät deshalb insbesondere dort unter Beschuss, wo sie die öffentlichen Kassen belastet. In Frage steht dabei nicht die präventive Vernunft als solche, sondern wer ihr Geltung verschaffen soll. Im Zuge der gegenwärtigen Ökonomisierung des Sozialen verwandelt sich der „Vorsorgestaat“ (Ewald 1993) zum ,aktivierenden Staat", der seine Bürger und Bürgerinnen aus der fürsorglichen Belagerung in die Freiheit der Selbstsorge entlässt und ihnen zumutet, ihre Lebensrisiken eigenverantwortlich zu managen. Nicht die pastorale Umsicht einer gleichermaßen behütenden wie kontrollierenden Verwaltung, sondern marktförmige Selbstregulation soll die optimale Allokation knapper Ressourcen gewährleisten und Risikominimierung mit Autonomiemaximierung verbinden. Prävention wird wichtiger denn je, aber sie wird zunehmend zur Sache der Individuen, die gehalten sind, sich selbst ökonomisch zu regieren. Wer sich als unternehmerisches Selbst behaupten will, tut gut daran, rechtzeitig ins eigene Humankapital zu investieren.

Und weil in dieser Logik jedes Handeln als Investition zu begreifen ist, sich aber eben auch als Fehlinvestition erweisen kann, muss das unternehmerische „Selbstverbesserungssubjekt" flexible Strategien der Fehlervermeidung sowie der Anpassung an veränderte Umweltbedingungen entwickeln. Unternehmer müssen Risiken eingehen, aber sie müssen auch alles tun, die Gewinn- und Verlustaussichten ihrer Entscheidungen soweit möglich zu kalkulieren. Kurzum, sie müssen kontinuierlich lernen und sind deshalb auf fortwährendes Feedback angewiesen. Das geht nicht allein, sondern nur in kontinuierlicher Auseinandersetzung mit anderen. Vorbeugen heißt zu kommunizieren und sich zu vernetzen. Auch hier gilt das Diktat des Komparativs: Je dichter die Kommunikation, je mehr Knoten das Netzwerk, desto geringer die verbleibenden Risiken.

Aktuelle Präventionsdiskurse ersetzen die traditionellen Mechanismen des Überwachens und Strafens deshalb durch ein Regime des Monitoring und freiwilliger Selbstkontrolle. Kompetenz- und Ressourcenorientierung lauten die Schlagworte, und nicht nur in der Suchtprävention hat sich inzwischen die Erkenntnis durchgesetzt, die Stärken zu stärken sei wirksamer, als Ängste zu schüren oder Verbote auszusprechen. Ohne Drohszenarien kommt indes auch der Appell an die Selbstverantwortung nicht aus: Wer es an Einsicht fehlen lässt und etwa auf Tabak oder Alkohol nicht verzichten will, wer keinen Sport treibt oder regelmäßige Vorsorgeuntersuchungen versäumt, der hat auch die Folgen selbst zu tragen - sei es in Form höherer Versicherungsprämien, sei es in Form von Erkrankungswahrscheinlichkeit und geringerer Lebensdauer. Je dichter das Netz präventiver 
Kontrollmöglichkeiten, desto fahrlässiger handelt, wer sie nicht wahrnimmt. Vorbeugung avanciert zur moralischen Pflicht, deren Unabweisbarkeit gerade darauf beruht, dass sie nicht an hehre Ideale, sondern an das Eigeninteresse appelliert. Weil dieses Gebot sich auf alle Lebensbereiche erstreckt, ist ihm eine ebenso universelle Schuldzuweisung eingeschrieben. Welche kleinen oder großen Katastrophen den Einzelnen auch ereilen mögen, in letzter Konsequenz sind sie stets ein Ergebnis seiner unzureichenden Sorge um sich. Dieser Schuld entgeht niemand, denn der Ausgang allen präventiven Bemühens steht immer schon fest: In the long run we are all dead. Vorbeugung gewährt allenfalls Aufschub. Vielleicht ist das der Grund für das konstitutiv schlechte Gewissen, dass Präventionisten haben - und anderen machen.

Wie könnte das Gesagte in ein Forschungsprogramm übersetzt werden? Dazu abschließend einige wenige Anmerkungen: Einer soziologischen Reflexion, die auf Problematisierung des vermeintlich Selbstverständlichen abzielt - und der Gemeinspruch, Vorbeugen sei besser als Heilen, besitzt zweifellos eine nahezu fraglose Plausibilität - kann es nicht darum gehen, die Vorbeugung gegenüber anderen Sozialtechnologien und Selbstpraktiken hochzuhalten oder aber zu verdammen oder bestimmte Präventionskonzepte gegen andere auszuspielen. Vielmehr läge die Aufgabe darin, die spezifische Rationalität sowie die Technologien der Vorbeugung herauszuarbeiten, die heterogenen Elemente und Ursprünge des Präventionsdispositivs und sein Diffundieren in die unterschiedlichsten gesellschaftlichen Bereiche nachzuzeichnen. Anders gesagt: Es ginge darum, Prävention als einen übergreifenden Modus des Zukunftsmanagements zeitgenössischer Gesellschaften herauszupräparieren. Um diesen Vergesellschaftungsmodus zu beschreiben, wäre eine systematische Darstellung der unterschiedlichen Präventionstypen und -strategien zu verbinden mit exemplarischen Fallstudien zu einzelnen Präventionsfeldern. $\mathrm{Zu}$ untersuchen wären nicht zuletzt die massenkulturellen Imaginationen und Phantasmen, die sich - wie in Spielbergs „Minority Report“ - an das Ideal der Vorbeugung heften. Gesucht wird eine Antwort auf die Frage: Was bedeutet es, wenn eine Gesellschaft sich die Losung von „Precrime“ zu eigen macht: „,.. nur ein Leben in Sicherheit garantiert auch ein Leben in Freiheit"?

\section{Bibliographie}

Billis, D. (1981) At risk of prevention. In: Journal of Social Policy, 10: 367-379.

Canguilhem, G. (1974) Neue Überlegungen zum Normalen und zum Pathologischen. In: Das Normale und das Pathologische. München: Hanser.

Caplan. G. (1964) Principles of Preventive Psychiatry. New York/London: Basic Books.

Castel, R. (1983) Von der Gefährlichkeit zum Risiko. In: Wambach, M. M. (Hg.) Der Mensch als Risiko. Zur Logik von Prävention und Früherkennung. Frankfurt/M.: Suhrkamp.

Dean, M. (1998) Risk, Calculable and Incalculable. In: Soziale Welt, 49: 25-42.

Defert, D. (1991) ,Popular life" and insurance technology. In: Burchell, G./Gordon, C./Miller, P. (eds.) The Foucault Effect. Studies in Governmentality. Chicago: University of Chicago Press.

Durkheim, E. (1895/1984) Die Regeln der soziologischen Methode. Frankfurt/M.: Suhrkamp.

Ewald, F. (1989) Die Versicherungs-Gesellschaft. In: Kritische Justiz, 22: 385-393. 
Ewald, F. (1991) Insurance and Risk. In: Burchell, G./Gordon, C./Miller, P. (eds.) The Foucault Effect. Studies in Governmentality. Chicago: University of Chicago Press.

Ewald, F. (1993) Der Vorsorgestaat. Frankfurt/M.: Suhrkamp.

Link, J. (1997) Versuch über den Normalismus, Opladen: Westdeutscher Verlag.

Luhmann, N. (1990) Risiko und Gefahr. In: Soziologische Aufklärung 5. Opladen: Westdeutscher Verlag.

Luhmann, N. (1991) Soziologie des Risikos, Berlin/New York: De Gruyter.

Makropoulos, M. (1990) Möglichkeitsbändigungen. Disziplin und Versicherung als Konzepte zur sozialen Steuerung von Kontingenz. In: Soziale Welt, 41: 407-423.

Matza, D. (1973) Abweichendes Verhalten. Heidelberg: Quelle und Meyer.

Popitz, H. (1968) Über die Präventivwirkung des Nichtwissens. Dunkelziffer, Norm und Strafe. Tübingen: Mohr und Siebeck.

Quetelet, A. (1869/1914/1921) Soziale Physik (186). 2. Bde. Jena: Fischer.

Reiwald, P. (1948) Verbrechensverhütung als Teil der Gesellschaftspsychohygiene. In: Meng, H. (Hg.) Die Prophylaxe des Verbrechens. Basel: Schwabe.

Wilson, J. W./Kelling, G. L. (1982) The police and neighborhood safety: Broken Windows. In: Atlantic Monthly, March: 29-39.

Ulrich Bröckling, Soziologe, ist Professor für Ethik, Politik, Rhetorik am Institut für Politikwissenschaft der Universität Leipzig. Lehr- und Forschungsschwerpunkte: Soziologie von Sozial- und Selbsttechnologien, Studies of Governmentality, Kultursoziologie, Schul- und Bildungssoziologie, Philosophische Anthropologie sowie die Soziologie des Kriegs und des Militärs.

E-mail: broeckling@uni-leipzig.de 